\title{
Quantitative Susceptibility Mapping in Ischemic Stroke Patients
}

Jasmin Probst ${ }^{1}$, Marco Piccirelli ${ }^{2}$, Athina Pangalu ${ }^{2}$, Andreas Deistung $^{3}$, Jan Klohs ${ }^{4}$, Andreas Luft ${ }^{1}$, Susanne Wegener ${ }^{1}$

${ }^{1}$ Department of Neurology, University Hospital Zurich and University of Zurich, Switzerland, ${ }^{2}$ Department of Neuroradiology, University Hospital Zurich, Switzerland, ${ }^{3}$ Medical Physics Group, Institute of Diagnostic and Interventional Radiology, University Hospital Jena, Germany, ${ }^{4}$ Institute for Biomedical Engineering, University and ETH Zurich, Switzerland

\section{Introduction}

Quantitative Susceptibility Mapping (QSM) is a novel post-processing method for gradient echo magnetic resonance imaging (MRI) (1). In acute stroke, it has shown that QSM is able to detect venous structures with increased susceptibility values in the ischemic hemisphere (2). QSM can help to estimate oxygen content of blood vessels, which is an important parameter in brain ischemia and reperfusion. Our goal was to analyze susceptibility signals of cortical veins in stroke patients to gather more information about the infarct and its collateral supply after stroke.

\section{Methods}

Data from 13 stroke patients with successfully recanalized middle cerebral artery (MCA)-M1 segment occlusion were collected.

MRI images acquired 24-72h after thrombectomy were analysed using ImageJ (3).

The following clinical variables were collected: age, sex, cardiovascular risk factors, stroke etiology, National Institute of Health Stroke Scale (NIHSS) on admission and stroke-to-needle time.

Outcome measures were: flow within the MCA-M1 after recanalization determined by duplex sonography, NIHSS after 24 hours, modified Rankin Scale (mRS) after 3 months, and infarct volume after recanalization measured on diffusion-weighted imaging (DWI) at 24-72h.

QSM signals of veins were explored and compared between stroke core, boundaries of the ipsilateral MCA territory and contralateral MCA territory using the nonparametric Wilcoxon signed-rank test.

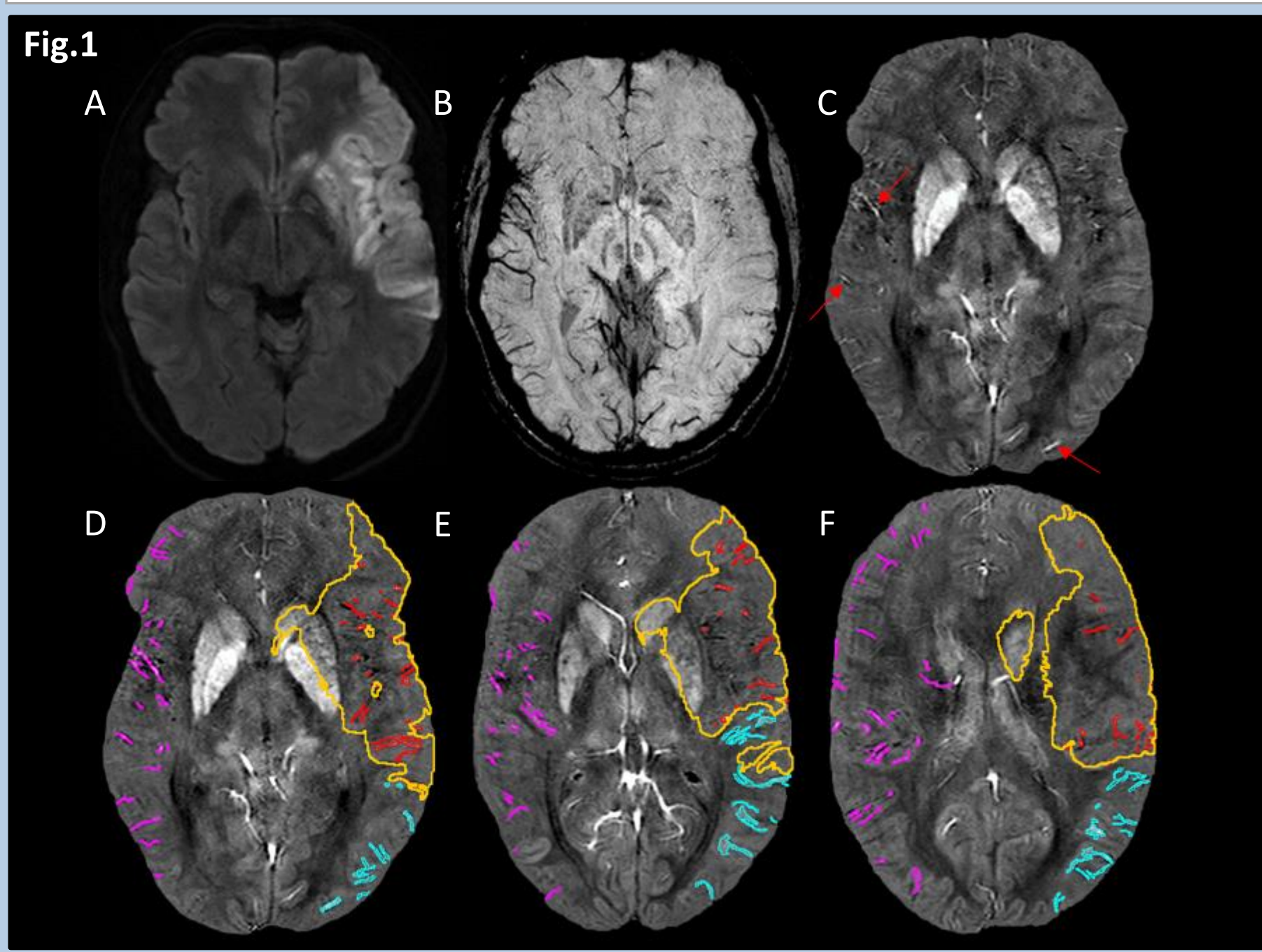

Fig.1) Demonstration of quantitative susceptibility maps of a stroke patient. The DWI (A) shows the lesion on the left ischemic hemisphere. (B) shows the susceptibility weighted image (SWI). The quantitative susceptibility map (QSM) is depicted in (C). Veins with high deoxyhemoglobin content show high susceptibility values on QSM (red arrows). (D-F) show cortical veins on QSM within the different territories; stroke in red, infarct border in cyan, contralateral MCA in magenta. The stroke territory is delineated in orange.

\section{Conclusions and Outlook}

After recanalization of MCA-M1 territory stroke, no prominent vessels with high susceptibility were found, but low venous susceptibility signal within vessels in the infarct area, likely caused by low deoxyhemoglobin content. This might indicate either normalized oxyhemoglobin levels after recanalization or poor oxygen extraction in the infarct based on tissue damage. Further exploration of the QSM signal and correlation with infarct size and outcome will reveal which of these processes underlie the observed signal changes and its prognostic value for stroke recovery.

\section{References:}

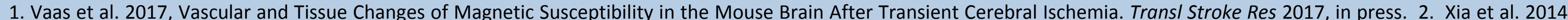

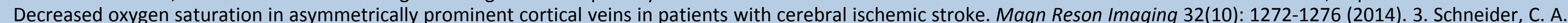
Rasband, W. S. \& Eliceiri, K. W., NIH Image to ImageJ: 25 years of image analysis, Nature methods 9(7): 671-675 (2012)

\section{Results}

Table 1: Patient characteristics

Stroke patients

$(n=13)$

\section{Demographic data}

Age, $y$ (range)

Female, $\mathrm{n}(\%)$

$68(45-87)$

Risk factors, $\mathbf{n}$ (\%)

Diabetes mellitus

Dyslipidemia

Smoking

Hypertension

Coronary heart disease

Atrial Fibrillation

Persistent foramen ovale 0

Prior stroke

Stroke etiology, $n(\%)$

Large-vessel disease

Cardioembolic

Small-artery disease

Other cause

Undetermined

\section{Stroke patients \\ Flow MCA-M1 [mmHg], median (IOR) \\ $(n=13)$ \\ Table 2:} Ipsilateral systolic

Contralateral systolic

Ipsilateral diastolic

Contralateral diastolic

Stroke-to-needle time [min],

median (IQR)

Clinical Scores, median (IQR)

NIHSS on admission

NIHSS after 24h

NIHSS difference

Infarct volume after

$109(85-141)$

$104(83-120)$

$43(30-52)$

$39(29-51)$

recanalization $\left[\mathrm{cm}^{3}\right]$, median (IQR) $25(12-83)$

(75-268) Evaluation after 3 months

with a median mRS of 1 . The

$8(4-12)$

$1(0-3)$

median infarct volume measured on DWI after recanalization was $25 \mathrm{~cm}^{3}$. One patient was excluded from the analysis due to artefacts on QSM images.

Fig.2

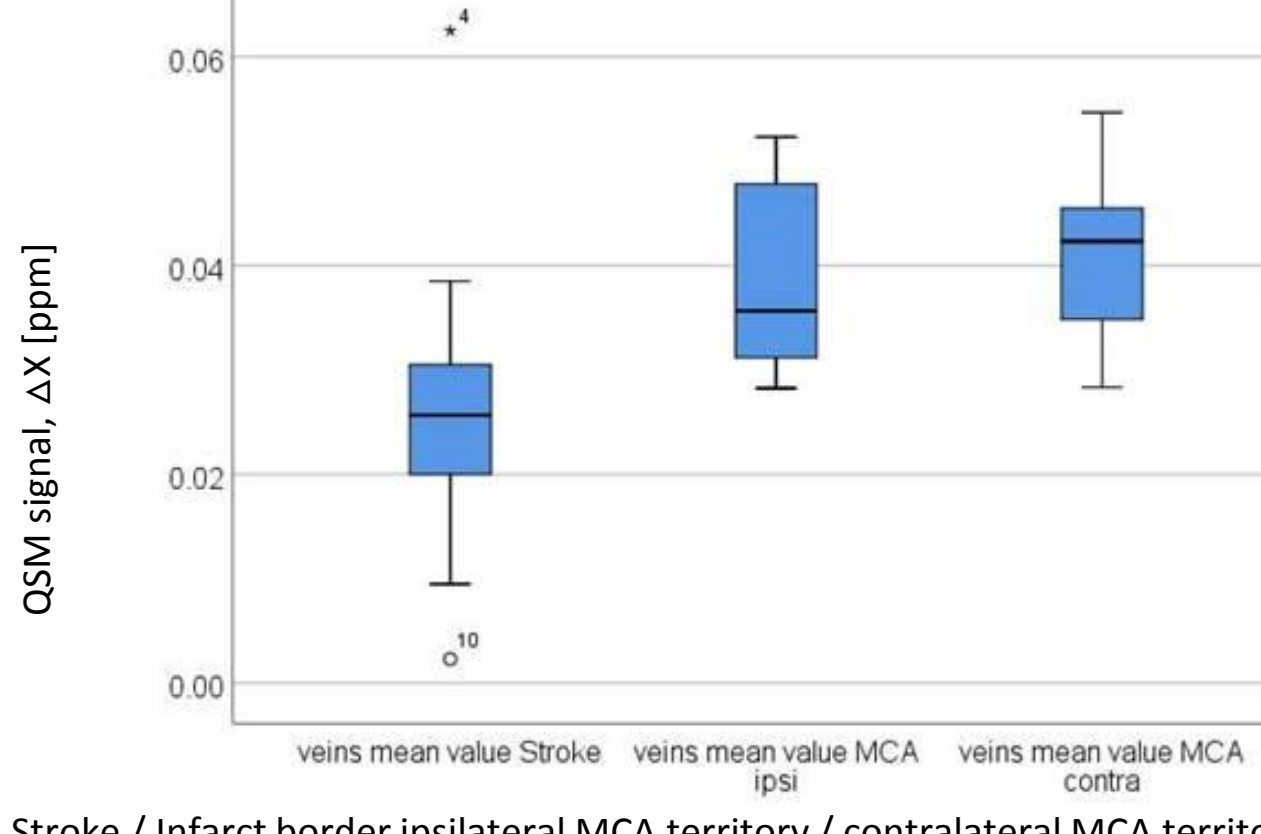

Table 3: QSM signals

\begin{tabular}{|c|c|c|c|}
\hline & Stroke territory & MCA ipsilateral & MCA contralateral \\
\hline
\end{tabular}

of veins,

median (IQR) $\quad 0.026(0.017-0.032)$

$0.042(0.034-0.047) \quad 0.013$

Fig.2 and Table 3) Comparison of the susceptibility of cortical veins show significantly reduced susceptibility signals within the stroke territory (median 0.026, IQR 0.017-0.032) compared to the infarct border (MCA ipsilateral, median 0.036, IQR 0.030-0.049), $p=0.013$. Furthermore, the susceptibility of the stroke territory is significantly lower than the signal on the control hemisphere (MCA contralateral, median 0.042, IQR 0.034-0.047), $p=0.013$. Note the similarity of QSM signals between the infarct border ipsilateral MCA territory and the contralateral MCA territory.
Table 2: Treatment and Outcome characteristics

mRS after 3 months 